\title{
Design of Facial Impact Protection Gear for Cyclists
}

\author{
Sanga Monthatipkul, Pio Iovenitti", Igor Sbarski \\ Faculty of Engineering \& Industrial Sciences, Swinburne University of Technology, Victoria, Australia \\ Email: "piovenitti@swin.edu.au
}

Received April 16, 2012; revised May 16, 2012; accepted June 2, 2012

\begin{abstract}
The concept of facial impact protection mask for cyclists is proposed in response to increased participation in cycling and the need for injury prevention. The research aims to develop an approach for design of facial impact protection gear to reduce the risk of severe injury. Impact test equipment and procedure, face surrogate and protection material performance criteria are developed. Three groups of protective materials - rigid crushable, semi rigid, and soft cushion foams are tested and assessed according to criteria. The criteria are linked to measures of the risk of facial and brain injuries: HIC (Head Injury Criterion), peak deceleration, Face-bone damage and energy absorption. The impact energy is simulated by a drop test using a $48 \mathrm{~mm}$-radius-steel hemispherical impactor, with a weight of $4.63 \mathrm{~kg}$ similar to that of headform J specified in AS/NZS standard. The drop-height is $1500 \mathrm{~mm}$, and the linear deceleration force of the impactor is recorded and used to establish the performance of the materials. The HIC is used to predict the risk of brain injury, whereas the developed face surrogate is used to assess facial bone injury. A $5.4 \mathrm{~m} / \mathrm{s}$ facial impact to the unprotected-face of a cyclist can result in the risk of severe facial bone fracture and mild brain injury. The impact test results for rigid foam protection of $40 \mathrm{~mm}$ thickness shows no densification (bottom out) and absorbs the impact energy without damage to the Foam-bone of the face surrogate. At $20 \mathrm{~mm}$ thickness, rigid polyurethane foams performed best with Foam-bone damage ranging from $15.1 \%$ to $20.5 \%$. Other materials with thicknesses of 20 to $28 \mathrm{~mm}$ showed Foam-bone damage between $21.8 \%$ and $35.1 \%$. The HIC values ranged from 267 to 522 , with memory foams and expanded polystyrene foam having the lowest values. Peak deceleration ranged from $71 \mathrm{~g}$ to $105 \mathrm{~g}$ for the materials tested. It is concluded that the impact energy can be dissipated by the protection material thereby reducing the risk of severe facial injury to the protected area.
\end{abstract}

Keywords: Facial Impact Protection; Cyclist; Energy Absorption; Impact Testing; Road Safety; Injury Prevention

\section{Introduction}

The increasing use of cycling for transportation has meant an increased number of persons at risk of an accident and severe facial injury caused by falls. Thomson et al. [1,2] and Harrison and Shepherd [3] report on severe facial injury associated with bicycle-related accidents and suggest the need for face protection. Facial injury may cause loss of function, loss of facial expression due to facial nerve damage, poor cosmesis, and loss of personal identity [4]. Facial fractures, especially in children, can lead to growth disturbances and condylar joint ankylosis $[5,6]$. Currently, the only head protection available is the bicycle helmet that aims to prevent brain injury due to an impact in a fall, while the face is left unprotected. Even though the trend in cycling is increasing, the development of additional safety equipment for cyclists has not been addressed, and hence, a facial impact protection mask for cyclists is proposed in this research.

A review of the literature has found no research on fa-

"Corresponding author. cial protection equipment for cyclists. Although, at least one helmet with chin guard is commercially available for mountain bike riding, there is no published research on this safety equipment's performance. The mountain bike helmet is commonly known as "full face helmet", which is a specialized helmet designed for sports activities. Such a helmet is heavy and weighs $\sim 1 \mathrm{~kg}$, whereas the typical bicycle helmet weighs $\sim(0.25-0.35) \mathrm{kg}$, although Australian standard [7] recommends a maximum of 0.5 $\mathrm{kg}$ for the helmet of headform size $\mathrm{J}$.

The present research foresees a concept design for a facial impact protection mask which is expected to be comfortable to wear and is of a flexible form to follow the face contours to reduce the risk of severe injury due to falls. A first step in the development of the facial mask is the establishment of test procedure and performance criteria, followed by testing of potential energy absorbing materials. For evaluation, the mask is considered as a flat shape, which represents the zygomas (nose, and left and right zygoma), and is subjected to impact tests. The overall aim is to develop an approach for the design of 
facial impact protection gear. An objective of the research is to test a range of potential and readily available materials to evaluate their ability to reduce damage to the developed face surrogate (reported in an earlier paper). The reduction in risk of brain injury will also be considered, as well as the energy absorption of the material. The objectives also include the design of the impactor for use in the drop tower and selection of performance criteria to evaluate the materials.

A concept design for the facial protection mask is shown in Figure 1. The commercially available "full face helmet" protects the lower-jaw bone and joints (mandible and mandibular condyle) by relying solely on the hard plastic shell to dissipate impact energy through its deflection and transmission to the head. The proposed design would use a liner and padding to crush and dissipate the impact energy.

\section{Background}

The following literature review includes test standards on bicycle helmets, protection materials, and performance criteria for evaluating impact protection materials.

\subsection{Test Standards}

A review of the literature has found no standard that covers facial impact protection equipment for cyclists. There are standards for helmets as protective equipment for cyclists, and these specify a test procedure for impact protection of the skull, which is usually represented by a rigid headform. However, since the present research focuses on facial impact, which is more fragile than the skull, the bio-fidelity of the test rig becomes an issue, which has been summarized by Hampton [8] and led to various developments of specialized face-compliant headform. While commercial face surrogates are available, they are expensive, and the large number of tests required for this research justified the development of an alternative deformable face surrogate having a bluntimpact-dynamic response comparable to the human face. A crushable "face surrogate" (representing the zygomas) was developed and used in the testing. The surrogate

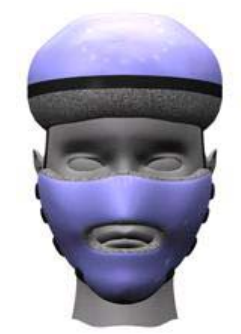

Front view

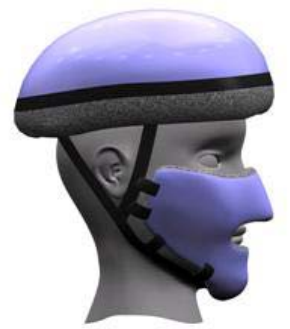

Side view

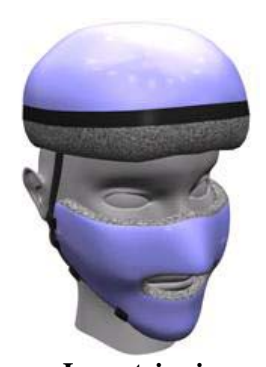

Isometric view
Figure 1. Concept design of the facial protection mask attached to the strap of the typical bicycle helmet. development was based on the work of Melvin et al. [9] and Perl et al. [10] that utilized the biomechanical data published by Nyquist et al. [11] and Allsop et al. [12]. The face surrogate is a flat sandwich of rubber skin, Foam-bone (30 mm thick, rigid extruded-polystyrene foam with a pattern of $6 \mathrm{~mm}$-diameter holes), and rubber backing. As has been pointed out by Hampson [8], the damage to the Foam-bone can also be used to assess risk of facial bone injury.

Since the shape of the facial bone is not as flat as that of the face surrogate, a focused contact force is used for the impact studies. To simulate this focused force, a hemispherical impactor was designed and built for use in this study. The design of the impactor is based on AS/ NZS standard [13], with the weight of medium-sized headform and the shape of the hemispherical steel anvil. Although actual impact surfaces on roads are likely to vary from mostly flat shapes to some with corner/wedge shapes, the spherical shaped impactor provides a representation in between these. The impactor is dropped on to the test samples, which consist of protection material over the face surrogate.

\subsection{Impact Protection Material}

The research on head impact protection is well documented [14-18]. These studies focus on using rigid crushable foam (foam liner) to manage the impact force and energy absorption. The test procedure is optimized for impact protection at the skull, which is represented by a rigid headform. The foam liner is then crushed by the headform and a rigid steel anvil. Foam with low stiffness will reach densification (bottom out) quickly, after which a high force will be generated. Consequently, a highly stiff foam liner with high density $\left(70-90 \mathrm{~kg} / \mathrm{m}^{3}\right)$ is needed in order to pass the test. Many researchers argue that the headform is much stiffer than the human skull, and suggest a lower density foam liner may have more benefits. Morgan and Szabo [19] incorporated lower density foam $\left(30-35 \mathrm{~kg} / \mathrm{m}^{3}\right)$ with higher density $(70-75$ $\mathrm{kg} / \mathrm{m}^{3}$ ) foam and demonstrated an improvement in performance, although the rigid headform is used in their study.

Since the stiffness of the facial bone is much less than that of the skull, a range of even lower density $(<30$ $\mathrm{kg} / \mathrm{m}^{3}$ ) expanded-polystyrene (EPS) foam liner seems possible, in addition to a range of semi-rigid foam and soft material. For example, EVA (Ethylene Vinyl Acetate) has some shock-absorption application in a mouth-guard and cricket helmet [20-23], and Polyurethane (PU) memory foam (slow-recovery PU foam) and flexible PU foam are used for pressure distribution in seat cushions [18]. Consequently, a range of these materials are evaluated in this study. 


\subsection{Performance Criteria}

The evaluation of performance involves a number of criteria relating to head injury and impact energy absorption of the protection material. In this study, the primary measure is the risk of brain injury as a result of blunt impact on the face. The secondary measure is the risk of facial injury. The last measure is the energy absorption capability of the protective material. The following sections present the literature review related to the three criteria.

\subsubsection{Brain Injury Risk Assessment}

In many countries, mandatory bicycle helmet wearing has been enacted, along with the requirement for the helmet to pass the impact testing specified in the standards, which aim to assess the risk of brain injury by measuring the level of linear deceleration of the head during the impact. A higher deceleration level typically causes higher risk of brain injury. Most standards impose a pass/fail criteria, for example, AS/NZS standard [7,13] requires 1500-mm-drop height of a rigid headform (2.5 $6.1 \mathrm{~kg}$ ) fastened with the helmet to be tested. To pass the test, the helmet crushing must not generate peak linear-deceleration force $\left(G_{\text {peak }}\right)$ greater than $250 \mathrm{~g}$, and the cumulative impact duration must not exceed $3 \mathrm{~ms}$ (for $G_{\text {peak }}>200 \mathrm{~g}$ ) or $6 \mathrm{~ms}$ (for $G_{\text {peak }}>150 \mathrm{~g}$ ). The inclusion of impact duration makes it difficult to compare performance between different protective materials, thus, the HIC (Head Injury Criterion) is used in this study.

HIC was introduced in 1972 by NHTSA (National Highway Traffic Safety Administration) in the USA [24] and has been widely used as a criterion to judge the risk of head and brain injury involving blunt impact on the head. McLean et al. [25] presented a summary of a critique and development of HIC, which links to the Wayne State Tolerance Curve (WSTC) that represents the relationship between acceleration, pulse duration, and intracranial pressure. It purports to describe the limit to cerebral concussion. Similar work was performed by the Japan Automobile Research Institute that developed a JARI Human Head Tolerance Curve based on impact studies on monkey skulls, and with a conversion scale to apply to the human head.

HIC incorporates impact duration in its definition (Equation (1)) and gives single a value that can be used to compare performance between protective materials.

$$
H I C=\max _{t_{1}, t_{2}}\left[\left(t_{2}-t_{1}\right)\left(\frac{1}{t_{2}-t_{1}} \int_{t_{1}}^{t_{2}} G(t) \mathrm{d} t\right)^{2.5}\right]
$$

$G(t)$ pulse of deceleration (in unit of $g$ ) as a function of time;

$t$ time in unit of second (s);

$t_{2}, t_{1}$ selected time under deceleration pulse that gave maximum $\mathrm{HIC}$;

$t_{2}-t_{1}$ selected pulse duration with limit of $3 \mathrm{~ms} \leq t_{2}-$ $t_{1} \leq 15 \mathrm{~ms}$.

In order to obtain the HIC value of an impact event, the Deceleration-Time history (deceleration in units of $g$ vs. time) of the head is applied as $G(t)$ in Equation (1). Head impact on different materials will usually have different HIC values. Higher value means a higher risk in severe brain injury as predicted in Table 1 [26].

Head impact that produces a higher HIC value than the limit is generally not acceptable. The typical HIC limit ranges from 700 to 1000 , depending on the application.

\subsubsection{Facial Injury Risk Assessment}

Research on skin penetration and laceration assessment can be found in forensic sciences and safety in the glass industry. However, these studies focus on ballistic impact and laceration caused by impact of a sharp object, as opposed to blunt-impact associated with the testing in this study. As a result, the facial skin injury cannot be assessed in this study. The data on facial bone tolerance are available [8,27,28], which are summarized in Table 2.

In this study, the face surrogate lacks a pressure sensitive device, and hence, pressure cannot be measured. Moreover, the peak force and peak deceleration can only be used if there is no damage to the Foam-bone, i.e., all impact energy is dissipated in the protection material. As

Table 1. HIC value and predicted injury [26].

\begin{tabular}{ll}
\hline HIC & Injury \\
\hline $135-519$ & Headache or dizziness, mild concussion \\
$520-899$ & Unconscious $<1$ hour, mild to severe concussion \\
$900-1254$ & Unconscious $1-6$ hours, severe concussion \\
$1255-1574$ & Unconscious $6-24$ hours, severe concussion \\
$1575-1859$ & Unconscious $>25$ hours, large haematoma \\
$>1860$ & Non survival \\
\hline
\end{tabular}

Table 2. Facial bone tolerance to impact force, peak pressure and peak deceleration.

\begin{tabular}{lccc}
\hline \multirow{2}{*}{ Facial bone } & \multicolumn{3}{c}{ Tolerance } \\
\cline { 2 - 4 } & $\begin{array}{c}\text { Peak force } \\
\text { (N) [8] }\end{array}$ & $\begin{array}{c}\text { Peak pressure } \\
\left(\mathbf{N} / \mathbf{m m}^{2}\right)\end{array}$ & $\begin{array}{c}\text { Peak deceleration } \\
(\mathbf{g})[\mathbf{2 7}, \mathbf{2 8}]\end{array}$ \\
\hline Nasal bone & $342-450$ & $0.13-0.34$ & 30 \\
Zygoma & $489-2401$ & $1.38-4.17$ & 50 \\
Zygomatic arch & $890-1779$ & $1.38-2.76$ & - \\
Maxilla & $668-1801$ & $1.03-2.07$ & 100 \\
Mandible & $685-1779$ & $2.76-6.20$ & 100 \\
Mandibular & - & - & 70 \\
angle & - & - & 200 \\
Orbital rim & - & & \\
\hline
\end{tabular}


a consequence, this study requires an additional parameter to assess the facial injury. The Foam-bone damage will be defined as the ratio between the Foam-bone crushing distance and the Foam-bone's original thickness. A lower value of Foam-bone damage is preferred as long as the HIC is below the limit.

\subsubsection{Energy Absorption}

The energy absorption $\left(E_{a b}\right)$ capability of the protective material is also an important parameter in head protection. A material with a high $E_{a b}$ returns little energy to the head, and hence, the head has a lower rebound velocity. Thus, the risk of severe secondary impact is reduced. McLean et al. [25] explain the preferred padding characteristics of energy absorbing material by analyzing the shape of Force-displacement curve, which is adapted for $E_{a b}$ analysis in this study. A material with a high $E_{a b}$ is desirable; however, no specific value of $E_{a b}$ as a criterion has been established.

\section{Methodology}

The following section outlines the impact test procedure and parameters, the experimental equipment and instrumentation, the materials tested, and the evaluation criteria: HIC limit, Foam-bone damage, and energy absorption.

\subsection{Impact Energy}

The impact energy was provided by dropping a steel hemispherical impactor (radius $48 \mathrm{~mm}$ ) in a drop tower shown in Figure 2. The mass of the impactor was 4.63 $\mathrm{kg}$, which was approximately the same as that of headform $J$ specified in AS/NZS standard [13]. The drop height was $1500 \mathrm{~mm}$, the impact energy was 68 Joules $(J)$ and the calculated impact velocity $\left(V_{i}\right)$ was $5.4 \mathrm{~m} / \mathrm{s}$.

\subsection{Data Acquisition System}

A charge type accelerometer (B \& K 4371) and an amplifier (B \& K Type 2635) were used to generate the acceleration signal, which was digitized by a digital oscilloscope (oscill_1, Tektronix TDS 2024) at the sampling rate of $50 \mathrm{kHz}$. The impact velocity $\left(V_{i}\right)$ was measured by two LED detectors spaced $100 \mathrm{~mm}$ apart, and the time was recorded by another oscilloscope (oscill_2, Tektronix TDS 210). Video of the impact event of test subject with a distance scale was recorded using a highspeed digital camera (Photron APX RS) at 3000 frames/ second with shutter speed of $1 / 15,000$ second. All data were then transferred to a personal computer for further analysis of the test results. Figure 2 shows the data acquisition system installed on the drop tower.

Equipment calibration was done by comparing dis- placement (double integral of acceleration) from accelerometer data with that of the high-speed video. Both results compared well especially during the loading phase. By using video data as reference, results from different impact velocities gave the average maximum error $\sim 2 \%$ during the loading phase, and $\sim 6 \%$ during the unloading phase.

\subsection{Test Samples}

The test samples were categorized into three groupsrigid crushable, semi rigid, and soft cushion materials. All materials were cut to size of $150 \mathrm{~mm} \times 150 \mathrm{~mm}$, and the thickness ranged from 20 to $40 \mathrm{~mm}$. Table 3 lists all candidate materials, as well as the face surrogate materials.

\subsection{Test Procedure}

A sample of the protective material on the face surrogate (Figure 3) is placed in the drop tower, and the impactor is dropped on the sample from a height of $1500 \mathrm{~mm}$. This procedure simulates an impact on the protected face. The impact velocity and deceleration $(g)$ versus time are recorded, and damage to the materials is observed. In some instances a high speed camera video recording of the impact was performed for verification of displacement and time. In addition, testing on the face surrogate without protection is performed in order to establish a reference. Tests were repeated 2 to 3 times with a new facebone each time. The number of samples tested was limited because of the long time required to produce the face-bone samples.

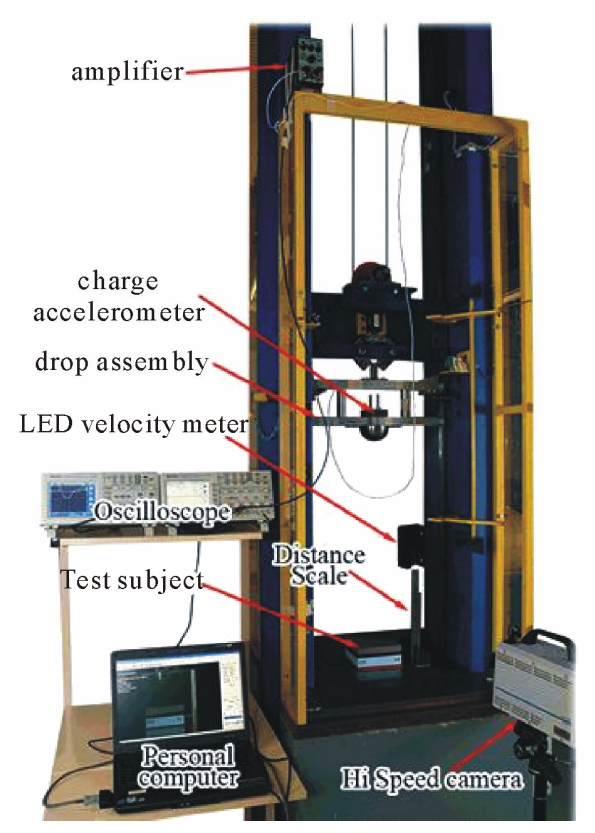

Figure 2. Drop tower and data acquisition system. 
Table 3. Energy absorbing materials tested and face surrogate.

\begin{tabular}{|c|c|c|c|c|}
\hline Material group & Material description & Measured density $\left(\mathrm{kg} / \mathrm{m}^{3}\right)$ & Thickness (mm) & Code (material-thickness) \\
\hline \multirow{6}{*}{ Rigid crushable foam } & \multirow{2}{*}{ Extruded polystyrene foam } & \multirow{2}{*}{39.1} & 40 & XPS40-40 \\
\hline & & & 20 & XPS40-20 \\
\hline & \multirow{2}{*}{ Rigid polyurethane foam } & \multirow{2}{*}{56.6} & 40 & PUR60-40 \\
\hline & & & 20 & PUR60-20 \\
\hline & \multirow{2}{*}{ Expanded polystyrene foam } & 21.0 & 25 & EPS21-25 \\
\hline & & 18.2 & 25 & EPS18-25 \\
\hline \multirow{3}{*}{ Semi rigid foam } & Polyethylene foam & 130.1 & 20 & PE130-20 \\
\hline & \multirow{2}{*}{ Ethylene vinyl acetate foam } & 137.9 & 20 & EVA140-20 \\
\hline & & 100.6 & 20 & EVA100-20 \\
\hline \multirow{3}{*}{ Soft cushion } & \multirow{2}{*}{ Polyurethane memory foam } & 104.0 & 28 & MEM120-28 \\
\hline & & 83.5 & 28 & MEM90-28 \\
\hline & Flexible polyurethane foam & 60.3 & 25 & PUf56-25 \\
\hline \multirow{3}{*}{$\begin{array}{l}\text { Face surrogate } \\
\text { (zygoma) }\end{array}$} & $\begin{array}{l}\text { Extruded polystyrene foam with } \\
6 \text {-mm hole pattern ( } 216 \text { holes })\end{array}$ & 28.5 & 30 & Foam-bone \\
\hline & Neoprene sponge & 145.5 & 10 & RS-10 \\
\hline & Abrasaflex rubber & 979.5 & 5 & AR-5 \\
\hline
\end{tabular}

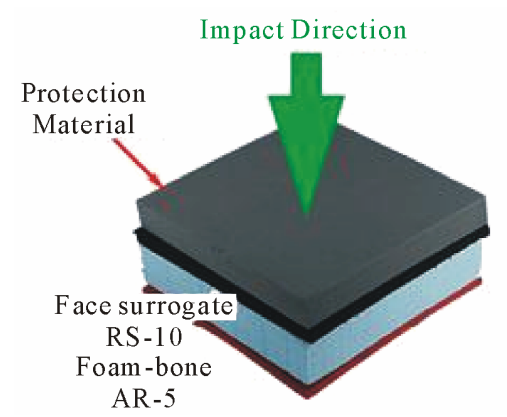

Figure 3. Impact on protected face.

\subsection{Evaluation Criteria}

There are four criteria for evaluation: the HIC limit for brain injury assessment, Foam-bone damage for facial injury assessment, peak deceleration for facial bone tolerance and energy absorption by the protection material.

\subsubsection{HIC Limit}

For brain injury assessment, a HIC limit of 1000 is normally used by researchers in the field. The HIC of each test was obtained by applying the HIC definition (Equation (1)) to the Deceleration-Time history. Protection with a lower HIC value was preferred, whereas a HIC value above 1000 was not acceptable.

\subsubsection{Peak Deceleration}

Peak deceleration tolerance is given in Table 2 and indi- cates the extent of facial bone injury.

\subsubsection{Foam-Bone Damage}

After the impact, the Foam-bone was observed for any damage. The Foam-bone damage was defined as the ratio between the Foam-bone indentation (crushing) distance and the Foam-bone's original thickness (Equation (2)). A lower value of Foam-bone damage is preferred as long as the HIC value is below the limit. Apart from indentation, fracture of the Foam-bone was also observed in some cases. Figure 4 illustrates the indentation and fracture as a result of direct impact on the surrogate (no protection).

Foam-bone damage

$$
=\frac{\text { Indentation Distance }}{\text { Foam-bone's original thickness }} \times 100 \%
$$

\subsubsection{Energy Absorption $\left(E_{a b}\right)$}

The absorption of energy at impact by the protection material reduces the HIC as well as reducing the impactor's rebound (bouncing) velocity, which can cause an additional risk of further injury through secondary head impact. Thus, the protection material with a higher $E_{a b}$ is considered better. The $E_{a b}$ value of a material can be illustrated by analyzing its Force-displacement curve as shown in Figure 5. The rigid impactor hitting the deformable material involves crushing (loading) and bouncing (unloading) phase. The contact force and crushing 


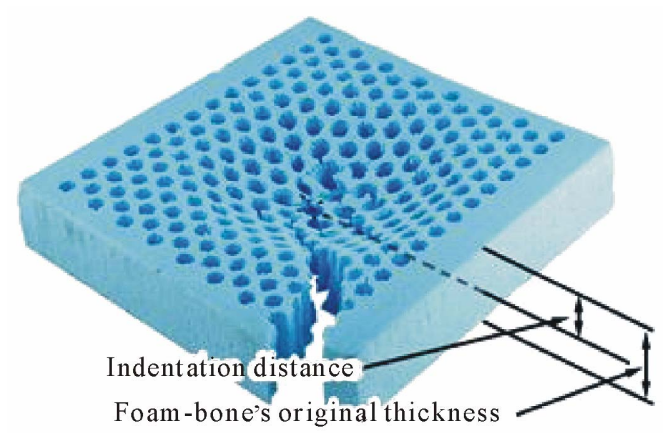

Figure 4. Illustration of Foam-bone damage.

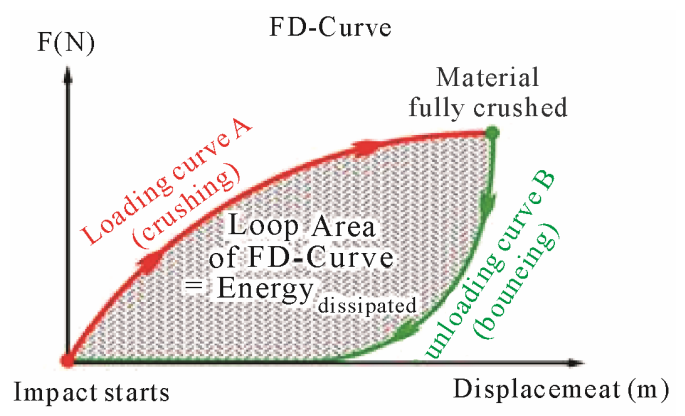

Figure 5. Energy from Force-displacement curve.

distance are represented by Loading curve $A$, whereas that of bouncing is represented by Unloading curve $B$. The energy of the impactor is the product of force by displacement, thus, the area under these curves represents the loading and unloading energy phase. If energy loss is negligible, the area under the Loading curve A represents the input energy (Energy (input $_{\text {int }}$, which is the same as the kinetic energy at the start of impact. The area under the Unloading curve $B$ represents the energy returning $\left(\right.$ Energy $\left._{\text {return }}\right)$ to the impactor. The energy dissipated during the impact event $\left(\right.$ Energy $\left._{\text {dissipated }}\right)$ is Energy input $_{\text {minus }}$

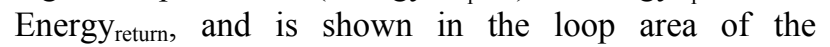
Force-displacement curve. The $E_{a b}$ value is then calculated by Equation (3).

$$
E_{a b}=\frac{\text { Energy }_{\text {dissipated }}}{\text { Energy }_{\text {input }}} \times 100 \%
$$

If the Foam-bone is not damaged, then the energy absorption is attributed wholly to crushing of the protection material. If both the protection material and Foam-bone are damaged, then energy absorption is shared between the Foam-bone and the protection material.

\section{Test Results and Discussion}

\subsection{Results of Impact on Protected Face}

The test results are listed in Table 4. These results are used to evaluate the performance of the protection materials at an impact speed $\left(V_{i}\right)$ of $5.4 \mathrm{~m} / \mathrm{s}$ (achieved through a drop height $1500 \mathrm{~mm}$ ) by impact testing on the protected face surrogate. The result of the unprotected face is also provided as reference. HIC, peak deceleration, energy absorption, and damage to the materials and face surrogate are also reported. Figure 6 shows the sample of the worst Foam-bone damage.

\subsection{Risk of Brain Injury Results}

The best materials are 40 mm-thick PUR60 and XPS40

Table 4. Impact test results on the protected face and reference unprotected face.

\begin{tabular}{|c|c|c|c|c|c|c|c|}
\hline Material group & $\begin{array}{c}\text { Protection } \\
\text { material }\end{array}$ & HIC & $\begin{array}{c}\text { Peak deceleration } \\
G_{\text {peak }}(\mathrm{g}) \\
\end{array}$ & $\begin{array}{c}\text { Foam-bone } \\
\text { damage }(\%)\end{array}$ & $E_{a b}(\%)$ & $\begin{array}{c}\text { Foam-bone } \\
\text { visible damage }\end{array}$ & $\begin{array}{c}\text { Protection material } \\
\text { visible damage }\end{array}$ \\
\hline \multirow{6}{*}{ Rigid } & XPS40-40 & 522 & 105 & 0.0 & 78.3 & None & Dented at top, bottom intact \\
\hline & XPS40-20 & 461 & 96 & 15.1 & 78.8 & Dented & Crushed, but no visible break \\
\hline & PUR60-40 & 276 & 74 & 0.0 & 88.4 & None & Radial break, crushed at centre \\
\hline & PUR60-20 & 365 & 83 & 20.5 & 85.7 & & Radial break, badly crushed centre \\
\hline & EPS21-25 & 267 & 71 & 24.0 & 83.0 & & \multirow[b]{2}{*}{ Crushed, circular break at centre } \\
\hline & EPS18-25 & 280 & 71 & 27.6 & 81.7 & & \\
\hline \multirow[b]{2}{*}{ Semi rigid } & PE130-20 & 337 & 83 & 21.8 & 88.2 & & \multirow{5}{*}{ Crushed, slow recovery, no damage } \\
\hline & EVA140-20 & 399 & 89 & 25.1 & 80.2 & Dented & \\
\hline \multirow{3}{*}{ Soft cushion } & MEM120-28 & 284 & 76 & 33.2 & 90.5 & & \\
\hline & MEM90-28 & 306 & 81 & 33.4 & 82.3 & & \\
\hline & PUf56-25 & 363 & 86 & 35.1 & 83.0 & & \\
\hline Foam-bone (no protection) & - & 453 & 112 & 48.7 & 85.1 & Dented, fractured & - \\
\hline
\end{tabular}




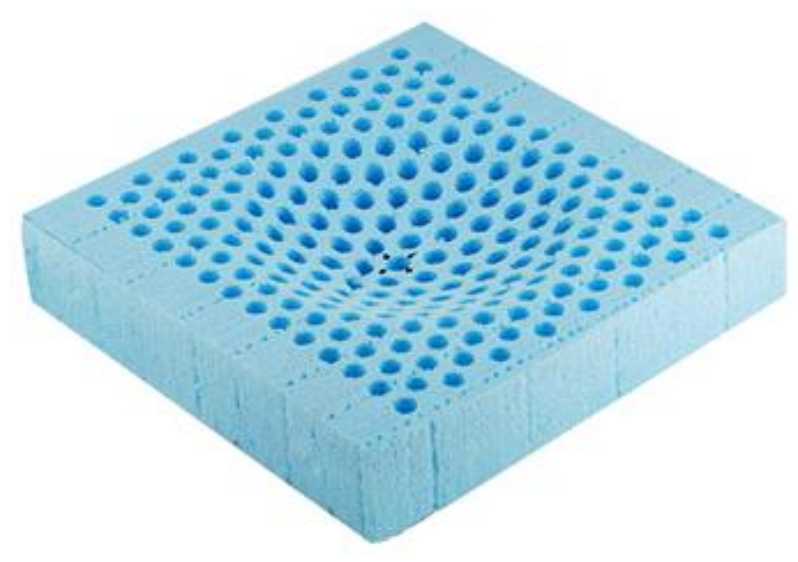

Figure 6. Sample of the worst Foam-bone damage (protected by PUf56-25) shows no fracture.

that completely protected the Foam-bone from damage. However, the corresponding HIC values are very different. This is because the PUR60 was severely damaged as a result of its low resistance to the impactor indentation. Consequently, the HIC is very low (276). On the other hand, the XPS40 was slightly damaged (dented at top) due to its high resistance to indentation that caused the HIC value to be very high (the highest in this study at 522). As a result, the PUR60-40 was considered the best protection material due to its superior performance in reducing risk of brain and facial injury. However, HIC values for all materials were well below the 1000 limit. For the other protection materials, the impact energy was not completely dissipated in the protection material and some energy was transferred to the face surrogate, hence, some damage to the Foam-bone was present. The combination of the protection and Foam-bone crushing caused the HIC values $(267-461)$ to stay within the mild concussion zone, as predicted in Table 1. Thus, the risk of brain injury was not significant.

\subsection{Risk of Facial Injury Results}

The Foam-bone damage was used to rank the face protection performance of each test, as listed in Table 4. The Foam-bone damage of $48.7 \%$, which was the case of no protection, was used as reference. The overall results showed that the rigid-crushable foam tended to perform better than the semi-rigid foam, whereas the soft-cushion foam exhibited poor performance. The best materials were PUR60-40 and XPS40-40, which showed zero Foam-bone damage ( $0.0 \%$ Foam-bone damage). The semi-rigid PE (21.8\% Foam-bone damage) showed exceptional performance that tended to compete with the rigid foams of the similar thickness. The worst material was the soft-cushion PUf56 that resulted in 35.1\% Foam-bone damage. However, the absence of "fracture" in the Foam-bone in all protection tests implied that the risk of facial bone injury had been significantly reduced.

In the case of zero Foam-bone damage, further assessment can be done by comparing the peak deceleration with the facial bone tolerance in Table 2. The PUR60-40 (peak deceleration $74 \mathrm{~g}$ ) provided protection to the maxilla, mandible, and mandibular angle, whereas the XPS40-40 (peak deceleration $105 \mathrm{~g}$ ) failed to protect most parts of the facial bone.

\subsection{Energy Absorption Performance}

In all tests, the hemispherical impactor generated a focused contact force that caused the protection material to fully crush before damaging the underlying face surrogate. An example of a video frame is shown in Figure 7.

When there is no face protection, the Foam-bone absorbed a large amount of impact energy $\left(85.1 \% E_{a b}\right)$ via its own crushing and fracture, whose illustration was in Figure 4. Adding a protection layer had varying effects on the $E_{a b}$ performance, which is discussed below.

In the case of zero Foam-bone damage, which was protected by $40 \mathrm{~mm}$-thick PUR60 and XPS40, all the impact energy was dissipated in the protection material. Thus, the $E_{a b}$ could be compared directly between the two. The results indicated that the PUR60 had a higher $E_{a b}$ than that of the XPS40 (88.4\% vs. $\left.78.3 \%\right)$, hence, PUR60 was considered better in $E_{a b}$ performance.

On the other hand, the other materials, which were unable to completely dissipate the impact energy, allowed some energy to transfer and damage the underlying Foam-bone. The combined crushing of the protection and the Foam-bone gave a total $E_{a b}$ that could only be compared within a group of similar Foam-bone damage, for example, MEM120 was better in $E_{a b}$ performance than MEM90 ( $E_{a b} 90.5 \%$ vs. $82.3 \%$ at the Foam-bone damage of $\sim 33 \%$ ). As a consequence, the overall $E_{a b}$ comparison could not be established in this study.

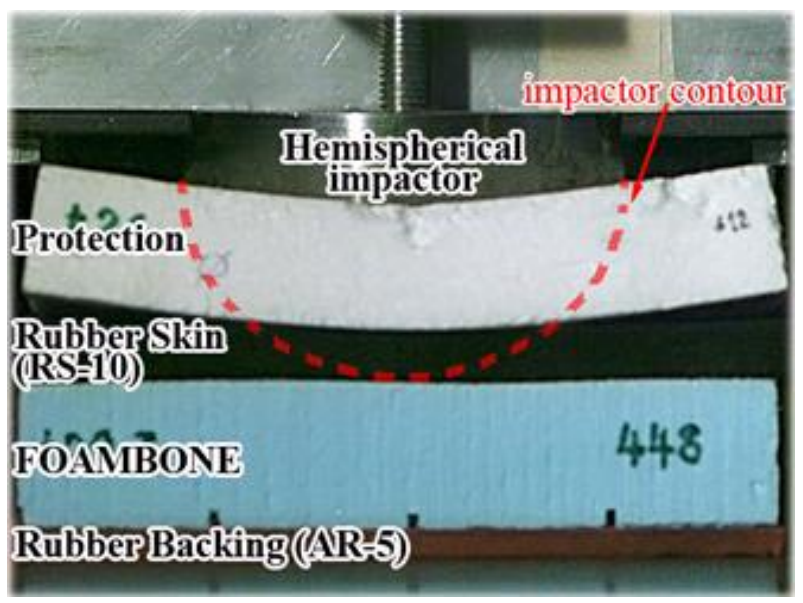

Figure 7. Video frame (at $6.6 \mathrm{~ms}$ ) shows the crushing of protection (EPS21-25) before crushing of Foam-bone. 


\subsection{Summary of Performance of Protection Materials}

This study tested a sandwich of protection material and frangible face surrogate, and hence, the $E_{a b}$ was the result of the combined crushing of both elements. Thus, the $E_{a b}$ comparison between protection materials could not easily be established. However, the damage to the face surrogate could be used to directly compare performance in facial bone injury reduction, whereas the HIC value could be used to assess the risk of brain injury.

Overall, all tests in this study showed HIC values well below the limit. Thus, the risk of brain injury was not significant. Nonetheless, in the case of no Foam-bone damage, a lower HIC value (e.g., PUR60-40) was preferred since it tended to further reduce the risk of brain injury. Moreover, the peak deceleration could be compared with the facial bone tolerance (peak deceleration in Table 2) for further assessment of facial bone injury risk.

When there is no face protection, the Foam-bone absorbed a large amount of impact energy via its own crushing and fracture. Adding a protection layer helped remove the "fracture" and reduce the Foam-bone damage, thus, it implied that the risk of facial bone injury had been significantly reduced.

The rigid-crushable foam of higher density PUR60 and XPS40 $\left(60 \mathrm{~kg} / \mathrm{m}^{3}\right.$ and $\left.40 \mathrm{~kg} / \mathrm{m}^{3}\right)$ showed better overall performance, which was in line with the headband study by Anderson et al. [29]. The performance moderately dropped with lower density EPS (21 and $18 \mathrm{~kg} / \mathrm{m}^{3}$ ). The semi-rigid PE130 foam showed exceptional performance that even surpassed some of rigid-crushable foams. Softcushion and lower density EVA foams (EVA100) exhibited poor performance and were not suitable for impact energy absorption. The slow-recovery feature of the PE and MEM foams suggested the materials could be reused, however, further tests are required to validate the performance for repeated use.

\section{Conclusions}

The research aims to develop an approach for the design of facial impact protection gear, and hence, impact test equipment and procedure are developed and potential materials are tested for energy absorption. The performance criteria for evaluating the mask are HIC value, peak deceleration, energy absorption and Foam-bone damage. Three groups of materials were tested, and the major findings are listed below:

- Adding a protection layer helps reduce the fracture and crushing of the face surrogate, thus, it implies that the risk of severe facial bone injury has been reduced.

- A $40 \mathrm{~mm}$-thick crushable foam provides more facial bone protection from risk of injury. However, it is expected that a thickness of this magnitude would most likely be used in some parts of the facial mask design requiring greater protection. At $20 \mathrm{~mm}$ thickness, rigid polyurethane foams performed best and reduced Foam-bone damage within $15.1 \%$ to $20.5 \%$. Other materials tested with thicknesses of 20 to 28 $\mathrm{mm}$ showed Foam-bone damage from $21.8 \%$ to $35.1 \%$.

- A $20 \mathrm{~mm}$-thick polyethylene foam (PE130-20) shows comparable performance to that of the rigid crushable foam of the similar thickness. PE's slow recovery property suggests it can be re-used.

- HIC values range from 267 to 522 , with the memory foam $(28 \mathrm{~mm})$ and expanded polystyrene foam $(25$ $\mathrm{mm}$ ) having the lowest values. Peak deceleration ranged from $71 \mathrm{~g}$ to $105 \mathrm{~g}$ for the materials tested.

In conclusion, the research is seen as a first step towards development of a facial impact protection gear for cyclists and a test procedure and performance criteria are proposed. A number of materials to absorb the impact energy are tested, and a concept design of a mask is also presented to illustrate its form and how it is worn.

\section{Acknowledgements}

The authors would like to thank the Swinburne FEIS technical support technical team (Warren Gooch, David Vass, and Meredith Jewson) for building the drop assembly, Stephen Guillow for technical information of the drop tower, Walter Chetcuti for setting up data acquisition system, and Horace Billon (Human Protection \& Performance Division, DSTO, Australian Government Department of Defence) for HIC programming.

\section{REFERENCES}

[1] D. C. Thompson, et al., "A Case-Control Study of the Effectiveness of Bicycle Safety Helmets in Preventing Facial Injury," American Journal of Public Health, Vol. 80, 1990, pp. 1471-1474. doi:10.2105/AJPH.80.12.1471

[2] D. C. Thompson, et al., "Effectiveness of Bicycle Safety Helmets in Preventing Serious Facial Injury," JamaJournal of the American Medical Association, Vol. 276, No. 24, 1996, pp. 1974-1975. doi:10.1001/jama.1996.03540240052030

[3] M. G. Harrison, and J. P. P. Shepherd, "The Circumstances and Scope for Prevention of Maxillofacial Injuries in Cyclists," Journal of the Royal College of Surgeons of Edinburgh, Vol. 44, No. 2, 1999, pp. 82-86.

[4] K. D. Parish and V. Cothran, "Facial Soft Tissue Injuries," 2006.

[5] D. B. Kim, M. Sacapano and R. A. Hardesty, "Facial Fractures in Children," Wright, Johnston and Mackenzie, Vol. 167, No. 2, 1997, p. 100.

[6] C. Lindqvist, et al., "Maxillofacial Fractures Sustained in Bicycle Accidents," International Journal of Oral and 
Maxillofacial Surgery, Vol. 15, No. 1, 1986, pp. 12-18. doi:10.1016/S0300-9785(86)80005-9

[7] Standards Australia and Standards New Zealand, "AS/ NZS 2063:2008, Bicycle Helmets," Australian/New Zealand Standard, 2008.

[8] D. Hampson, "Facial Injury: A Review of Biomechanical Studies and Test Procedures for Facial Injury Assessment," Journal of Biomechanics, Vol. 28, No. 1, 1995, pp. 1-7. doi:10.1016/0021-9290(95)80001-8

[9] J. W. Melvin, et al., "A Biomechanical Face for the Hybrid III Dummy," Stapp Car Crash Conference Proceedings, Vol. 39, 1995, pp. 140-151.

[10] T. R. Perl, et al., "Deformable Load Sensing Hybrid III Face," Stapp Car Crash Conference Proceedings, Vol. 33, 1989, pp. 29-42. doi: 10.4271/892427

[11] G. W. Nyquist, et al., "Facial Impact Tolerance and Response," Stapp Car Crash Conference Proceedings, Vol. 30, 1986, pp. 379-400.

[12] D. L. Allsop, et al., "Facial Impact Response-A Comparison of the Hybrid III Dummy and Human Cadaver," Stapp Car Crash Conference Proceedings, Vol. 32, 1988, pp. 139-155.

[13] Standards Australia and Standards New Zealand, "AS/ NZS 2512.3.1:2007, Methods of Testing Protective Helmets, Method 3.1: Determination of Impact Energy Attenuation-Helmet Drop Test," Australian/New Zealand Standard, 2007.

[14] R. Anderson, et al., "Further Development of a Protective Headband for Car Occupants," Australian Transport Safety Bureau, 2001.

[15] M. Henderson, "The Effectiveness of Bicycle Helmets: A Review," Bicycle Helmet Safety Institute, 1995.

[16] A. Gale and N. J. Mills, "Effect of Polystyrene Foam Liner Density on Motorcycle Helmet Shock Absorption," Plastics and Rubber Processing and Applications, Vol. 5, No. 2, 1985, pp. 101-108.

[17] A. L. Yettram, et al., "Materials for Motorcycle Crash Helmets-A Finite Element Parametric Study," Plastics and Rubber Processing and Applications, Vol. 22, No. 4, 1994, pp. 215-221.

[18] N. J. Mills, et al., "Polymer Foams for Personal Protec- tion: Cushions, Shoes and Helmets," Composites Science and Technology, Vol. 63, No. 16, 2003, pp. 2389-2400. doi:10.1016/S0266-3538(03)00272-0

[19] D. E. Morgan and L. S. Szabo, "Improved Shock Absorbing Liner for Helmets," Australian Transport Safety Bureau, 2001.

[20] R. A. Stretch, "The Impact Absorption Characteristics of Cricket Batting Helmets," Journal of Sports Sciences, Vol. 18, No. 12, 2000, pp. 959-964. doi:10.1080/026404100446766

[21] T. Takeda, et al., "The Influence of Impact Object Characteristics on Impact Force and Force Absorption by Mouthguard Material," Dental Traumatology, Vol. 20, No. 1, 2004, pp. 12-20. doi:10.1111/j.1600-4469.2004.00210.x

[22] N. K. Cummins and I. R. Spears, "The Effect of Mouthguard Design on Stresses in the Tooth-Bone Complex," Medicine \& Science in Sports \& Exercise, Vol. 34, No. 6, 2002, pp. 942-947. doi:10.1097/00005768-200206000-00006

[23] N. T. Paterson, et al., "A Finite Element Study of the Mechanics of Sports Mouthguards," Sports Engineering, Vol. 7, No. 4, 2004, pp. 182-195.

[24] B. G. McHenry, "Head Injury Criterion and the ATB," ATB Users' Group, 2004.

[25] A. J. McLean, et al., "Prevention of Head Injuries to Car Occupants: An Investigation of Interior Padding Options," University of Adelaide, 1997.

[26] D. Tyrell, K. Severson and B. Marguis, "Passenger Train Crashworthiness Studies," US Department of Transportation, 1995

[27] T. J. Rupp, M. Bednar and S. Karageanes, "Facial Fractures," 2011 http://emedicine.medscape.com/article/84613-overview

[28] K. F. Lee, et al., "The Impact-Absorbing Effects of Facial Fractures in Closed-Head Injuries," Journal of Neurosurgery, Vol. 66, No. 4, 1987, pp. 542-547. doi:10.3171/jns.1987.66.4.0542

[29] R. Anderson, G. Ponte and L. Streeter, "Development of Head Protection for Car Occupants," University of Adelaide, 2002. 